\title{
The energy-momentum tensor of spin-1 hadrons: formalism
}

\author{
Wim Cosyn ${ }^{1, a}$, Sabrina Cotogno ${ }^{2, b} \mathbb{C}$, Adam Freese $^{3, \mathrm{c}}$, Cédric Lorcéé $^{2, \mathrm{~d}}$ \\ ${ }^{1}$ Department of Physics and Astronomy, Ghent University, Proeftuinstraat 86, 9000 Ghent, Belgium \\ ${ }^{2}$ Centre de Physique Théorique, École polytechnique, CNRS, Université Paris-Saclay, 91128 Palaiseau, France \\ ${ }^{3}$ Argonne National Laboratory, Lemont, IL 60439, USA
}

Received: 20 March 2019 / Accepted: 23 May 2019 / Published online: 5 June 2019

(C) The Author(s) 2019

\begin{abstract}
We provide the complete decomposition of the local gauge-invariant energy-momentum tensor for spin-1 hadrons, including non-conserved terms for the individual parton flavors and antisymmetric contributions originating from intrinsic spin. We state sum rules for the gravitational form factors appearing in this decomposition and provide relations for the mass decomposition, work balance, total and orbital angular momentum, mass radius, and inertia tensor. Generalizing earlier work, we derive relations between the total and orbital angular momentum and the Mellin moments of twist- 2 and 3 generalized parton distributions, accessible in hard exclusive processes with spin-1 targets. Throughout the work, we comment on the unique features in these relations originating from the spin-1 nature of the hadron, being absent in the lower spin cases.
\end{abstract}

\section{Introduction}

In recent years, a lot of attention has been given to the energymomentum tensor (EMT) as a fundamental object of study in hadronic physics and QCD, see e.g. [1,2] and references therein. Hadronic matrix elements of the (local) EMT operator for quarks and gluons are parametrized in terms of gravitational form factors (GFFs), ${ }^{1}$ just as hadronic matrix elements

\footnotetext{
1 We call the form factors "gravitational" since the EMT is usually understood as the source of gravitational interactions. However, we do not measure in practice these form factors through gravity and we do not know the exact form that a theory of quantum gravity will take. Thus, it is unclear whether gravitation sees the symmetric, Belinfante-improved EMT as in general relativity or an asymmetric EMT as in EinsteinCartan theory [3]. Despite this, we refer to all form factors appearing in either the symmetric or asymmetric EMT as "gravitational" form factors.
}

\footnotetext{
a e-mail: wim.cosyn@ugent.be

b e-mail: sabrina.cotogno@polytechnique.edu

c e-mail: afreese@anl.gov

de-mail: cedric.lorce@polytechnique.edu
}

of the charge current operator are parametrized in terms of electromagnetic form factors. The GFFs encode properties that are of great interest, such as the hadronic mass and angular momentum sum rules and their spatial distributions $[1,4$ 6], and can shed light on novel properties of hadrons, such as the way that stress and shear forces are distributed within them $[2,4,7,8]$. The topic places itself in the more general quest for a thorough understanding of the hadron structure, in which the spatial and momentum distributions of quarks and gluons play a crucial role.

Much of the recent literature on the hadron structure and QCD EMT has focused on either spin-1/2 [2,5,6,8-10] or spin-0 [11-13] systems, see also [14-18] for recent lattice studies. In the former case, the proton has been the predominant object of study. This is natural, as nucleons are often understood as the primary building blocks of nuclear matter, and understanding the mass and spin decomposition of the proton is a necessary step in understanding the origin of most visible mass. In the latter case, the pion is of great interest not only for the unique role it plays in dynamical chiral symmetry breaking, which is believed to be the origin of the majority of hadron mass, but also because of its simplicity as a system and the ability to study its properties (including components of its EMT) on the lattice [19].

On the other hand, the hadronic physics community has in general dedicated little attention to the internal structure of hadrons of spin higher than 1/2. From a theoretical point of view, a full picture of higher spin hadrons and nuclei is desirable because it would serve in elucidating QCD dynamics: spin-1 (and higher spin) systems carry information on non-nucleonic degrees of freedom, i.e. the dynamics beyond quarks and gluons confined within the individual nucleons [20].

Information on spin-1 hadrons would allow us to thoroughly study such different parton contributions and dynamics in the spirit, for instance, of the theoretical calculations of the gravitational form factors for vector mesons in holo- 
graphic QCD [21] and on the lattice [22]. Being nearly the only experimentally available hadronic spin- 1 target, the deuteron has attracted a fair amount of attention over the past decades. It is the simplest bound state of more than one nucleon and, therefore, it has been of prime importance to unravel the nature of the nuclear binding. On the other hand, its internal structure and dynamics are the ultimate effect of the interactions between the elementary constituents, and this makes the deuteron a promising avenue towards understanding how QCD produces the force that binds nucleons together in nuclei [23]. After the first measurement by the HERMES collaboration of a tensor polarized collinear structure function of the deuteron [24], the so-called $b_{1}$ function defined in [25], it became clearer that going beyond the single-nucleon formulation is needed to describe the experimental data, especially in specific regions of the parton momentum range [26-30].

The same arguments hold for the study of the gluonic content of higher spin hadrons, which requires once again to account for additional gluon functions in momentum and coordinate space that are exclusive to tensor polarized structures and therefore related to spin- 1 or higher. This fact has stimulated a recent interest in the theoretical $[31,32]$ and lattice community [33,34]. The deuteron is thus expected to play a major role in the $12 \mathrm{GeV}$ program at Jefferson Lab (JLab) dedicated to spin-1 targets [35].

As a fundamental entity encoding the spatial and mechanical properties of hadrons, the EMT of a spin-1 system such as the deuteron contains much of this dynamical information that is of interest to the nuclear physics community. This information is encoded in GFFs familiar from the EMT of spin- 0 and spin- $1 / 2$ systems, but also within a host of additional form factors novel to spin-1 systems. This is analogous to the spin-1 electromagnetic current containing one more form factor than the spin-1/2 current, and has a similar origin. A spin-1 system has an additional degree of freedom, which can manifest itself in higher multipole moments (in this case, a quadrupole moment) or a tensor polarization mode. A full understanding of spin-1 systems requires a complete categorization of all the independent Lorentz structures that can appear in its EMT, and an elucidation of the physical significance of the GFFs that appear with these structures.

Expressions for the decomposition of the EMT for spin1 hadrons have appeared earlier in Refs. [21,36,37]. In the present work, we provide the complete EMT decomposition that also includes all non-conserved terms (appearing incompletely in [37]) and we study the properties of and the relations between the GFFs that parametrize the local operator for the EMT. More specifically, we derive new sum rules and present expressions for the mass and angular momentum decomposition of a spin-1 hadron in terms of the new structures for quarks and gluons.
The results in this paper may be relevant for the experiments at JLab and a future EIC [38] and for the proposed fixed-target projects @ LHC [39], where different polarized hadrons and nuclei can be employed. Current data for spin-1 tomography is rather scarce, with HERMES having measured deeply virtual Compton scattering (DVCS) on the deuteron with both unpolarized [40] and polarized targets [41]. In these measurements, hadrons were not detected in the final state, but simulations were used to select a sample of enhanced coherent deuteron contribution. More recently, Jefferson Lab has measured deeply virtual $\pi^{0}$ production on the deuteron [42] and a recent letter of intent allows for coherent deuteron DVCS measurements [43]. The latter should also be possible in Hall B using the ALERT detector [44]. Finally, generalized distribution amplitudes (GDAs) for the rho-rho meson pair, accessible in the crossed reaction $\gamma^{*} \gamma \rightarrow \rho \rho$ [45-47], can be related to the rho-meson GFFs similarly to the pion case [12] and could also potentially be studied at Belle II.

This work is organized as follows. In Sect. 2, we give a full decomposition of the most general form that the EMT of a spin-1 hadron can take. This section also contains sum rules that follow immediately from energy-momentum conservation. In Sect. 3, we calculate the multipole moments of matrix elements of the spin-1 EMT. Mass and angular momentum decompositions are derived in this section, along with additional sum rules and a work-energy balance relation. Section 4 explores the connections between the EMT and Mellin moments of twist- 2 and twist- 3 generalized parton distributions. Finally, in Sect. 5, we summarize our results. In addition, in Appendix A the form factors counting technique is reviewed, Appendix B and C contain additional information on Lorentz projectors and the polarization bilinears useful to obtain the parametrizations of the EMT, and Appendix D displays the covariant parametrization of the GPD correlator.

\section{Decomposition of the energy-momentum tensor}

The goal of this section is to construct the most general possible parametrization for the EMT of an on-shell, spin1 hadron in terms of GFFs. A variety of definitions exists for the EMT in QCD (for a review, see [1]), but here we work with the gauge-invariant kinetic form of the QCD EMT $T^{\mu \nu}=T_{q}^{\mu \nu}+T_{g}^{\mu \nu}$, where

$$
\begin{aligned}
T_{q}^{\mu \nu} & =\frac{1}{2} \bar{\psi} \gamma^{\mu} i \stackrel{\leftrightarrow}{D^{v}} \psi-g^{\mu \nu} \bar{\psi}\left(\frac{i}{2} \stackrel{\leftrightarrow}{\not D}-m\right) \psi, \\
T_{g}^{\mu \nu} & =-2 \operatorname{Tr}\left[F^{\mu \lambda} F_{\lambda}^{\nu}\right]+\frac{1}{2} g^{\mu \nu} \operatorname{Tr}\left[F^{\alpha \beta} F_{\alpha \beta}\right]
\end{aligned}
$$

with $\stackrel{\leftrightarrow}{D^{\mu}}=\left(\overrightarrow{\partial^{\mu}}-\overleftarrow{\partial^{\mu}}\right)-2 i g A^{\mu}$. Due to the presence of spin, the QCD EMT is in general not symmetric under exchange of 
its free Lorentz indices, with the entirety of the asymmetry in the quark contribution. The EMT $T^{\mu \nu}$ is a conserved current, with the symmetric and antisymmetric components being separately conserved. Accordingly, we consider the general form of the EMT in two layers: the symmetric component of the EMT and the full asymmetric EMT.

\subsection{Symmetric EMT}

For a spin-1 system, there are only six possible independent rank-2 Lorentz structures that are symmetric, P-even, T-even, consistent with the hermiticity property, Lorentzcovariant, linear in each of the initial and final state polarization vectors, and conserved [21,36]. This is fewer than the seven Lorentz structures that arise from the $(1,1)$ representation of the Lorentz group (see Appendix A), meaning one of those Lorentz structures is non-conserved. The conserved symmetric EMT takes the following form

$$
\begin{aligned}
\left\langle p^{\prime},\right. & \left.\lambda^{\prime}\left|T_{\mu \nu}(0)\right| p, \lambda\right\rangle \\
= & -2 P_{\mu} P_{\nu}\left[\left(\epsilon^{\prime *} \epsilon\right) \mathcal{G}_{1}(t)-\frac{\left(\Delta \epsilon^{*}\right)(\Delta \epsilon)}{2 M^{2}} \mathcal{G}_{2}(t)\right] \\
& -\frac{1}{2}\left(\Delta_{\mu} \Delta_{v}-\Delta^{2} g_{\mu \nu}\right)\left[\left(\epsilon^{\prime *} \epsilon\right) \mathcal{G}_{3}(t)-\frac{\left(\Delta \epsilon^{\prime *}\right)(\Delta \epsilon)}{2 M^{2}} \mathcal{G}_{4}(t)\right] \\
& +P_{\{\mu}\left(\epsilon_{\nu\}}^{\prime *}(\Delta \epsilon)-\epsilon_{\nu\}}\left(\Delta \epsilon^{\prime *}\right)\right) \mathcal{G}_{5}(t) \\
& +\frac{1}{2}\left[\Delta_{\{\mu}\left(\epsilon_{\nu\}}^{\prime *}(\Delta \epsilon)+\epsilon_{\nu\}}\left(\Delta \epsilon^{* *}\right)\right)\right. \\
& \left.-\epsilon_{\{\mu}^{\prime *} \epsilon_{\nu\}} \Delta^{2}-g_{\mu \nu}\left(\Delta \epsilon^{\prime *}\right)(\Delta \epsilon)\right] \mathcal{G}_{6}(t)
\end{aligned}
$$

where $M$ is the hadron mass, $P=\left(p^{\prime}+p\right) / 2$ is the average four-momentum, $t=\Delta^{2}$ with $\Delta=p^{\prime}-p$ is the fourmomentum transfer, and for each four-vector $a, b$ one has $a_{\{\mu} b_{\nu\}}=\left(a_{\mu} b_{\nu}+a_{\nu} b_{\mu}\right) / 2$. Energy and momentum must be conserved in a closed system, so in this decomposition of the symmetric EMT a sum over all partons is understood. The partial EMT for quarks and gluons does not have to be conserved however, so there are three additional independent Lorentz structures that can appear

$$
\begin{aligned}
\left\langle p^{\prime},\right. & \left.\lambda^{\prime}\left|T_{\mu \nu}^{a}(0)\right| p, \lambda\right\rangle \\
= & -2 P_{\mu} P_{\nu}\left[\left(\epsilon^{* *} \epsilon\right) \mathcal{G}_{1}^{a}(t)-\frac{\left(\Delta \epsilon^{\prime *}\right)(\Delta \epsilon)}{2 M^{2}} \mathcal{G}_{2}^{a}(t)\right] \\
& -\frac{1}{2}\left(\Delta_{\mu} \Delta_{\nu}-\Delta^{2} g_{\mu \nu}\right)\left[\left(\epsilon^{\prime *} \epsilon\right) \mathcal{G}_{3}^{a}(t)-\frac{\left(\Delta \epsilon^{* *}\right)(\Delta \epsilon)}{2 M^{2}} \mathcal{G}_{4}^{a}(t)\right] \\
& +P_{\{\mu}\left(\epsilon_{\nu\}}^{\prime *}(\Delta \epsilon)-\epsilon_{\nu\}}\left(\Delta \epsilon^{\prime *}\right)\right) \mathcal{G}_{5}^{a}(t) \\
& +\frac{1}{2}\left[\Delta_{\{\mu}\left(\epsilon_{\nu\}}^{*}(\Delta \epsilon)+\epsilon_{\nu\}}\left(\Delta \epsilon^{\prime *}\right)\right)\right. \\
& \left.-\epsilon_{\{\mu}^{\prime *} \epsilon_{\nu\}} \Delta^{2}-g_{\mu \nu}\left(\Delta \epsilon^{\prime *}\right)(\Delta \epsilon)\right] \mathcal{G}_{6}^{a}(t)
\end{aligned}
$$

$$
\begin{aligned}
& +\epsilon_{\{\mu}^{*} \epsilon_{\nu\}} M^{2} \mathcal{G}_{7}^{a}(t)+g_{\mu \nu} M^{2}\left(\epsilon^{\prime *} \epsilon\right) \mathcal{G}_{8}^{a}(t) \\
& +\frac{1}{2} g_{\mu \nu}\left(\Delta \epsilon^{\prime *}\right)(\Delta \epsilon) \mathcal{G}_{9}^{a}(t)
\end{aligned}
$$

where $a=q, g$. Summing over all partons, we should recover (2) which implies the following sum rules

$\sum_{a=q, g} \mathcal{G}_{i}^{a}(t)=0$ for $i=7,8,9$

Note that we have named the GFFs to agree with the conventions in [37], although we find an additional non-conserved pure trace GFF, in agreement with the counting in Appendix A. It should also be noted that unlike the total GFFs $\mathcal{G}_{i}(t)$, the partial GFFs $\mathcal{G}_{i}^{a}(t)$ are usually scale and scheme dependent.

\subsection{Asymmetric EMT}

When the constituents of a system possess intrinsic angular momentum, the EMT is in general expected to be asymmetric, see e.g. [1,48,49] for recent discussions. This is a simple consequence of the conservation of the generalized angular momentum $\partial_{\mu} M^{\mu \alpha \beta}=0$, with $M^{\mu \alpha \beta}=x^{\alpha} T^{\mu \beta}-x^{\beta} T^{\mu \alpha}+$ $S^{\mu \alpha \beta}$ and where $S^{\mu \alpha \beta}$ is the intrinsic generalized angular momentum tensor, which when combined with the conservation of the EMT implies that $T^{\alpha \beta}-T^{\beta \alpha}=-\partial_{\mu} S^{\mu \alpha \beta}$. In agreement with the counting in Appendix A, we find only two antisymmetric Lorentz structures satisfying all the constraints. The most general form of the EMT is therefore

$$
\begin{aligned}
\left\langle p^{\prime},\right. & \left.\lambda^{\prime}\left|T_{\mu \nu}^{a}(0)\right| p, \lambda\right\rangle \\
= & -2 P_{\mu} P_{\nu}\left[\left(\epsilon^{\prime *} \epsilon\right) \mathcal{G}_{1}^{a}(t)-\frac{\left(\Delta \epsilon^{\prime *}\right)(\Delta \epsilon)}{2 M^{2}} \mathcal{G}_{2}^{a}(t)\right] \\
& -\frac{1}{2}\left(\Delta_{\mu} \Delta_{\nu}-\Delta^{2} g_{\mu \nu}\right)\left[\left(\epsilon^{\prime *} \epsilon\right) \mathcal{G}_{3}^{a}(t)-\frac{\left(\Delta \epsilon^{\prime *}\right)(\Delta \epsilon)}{2 M^{2}} \mathcal{G}_{4}^{a}(t)\right] \\
& +P_{\{\mu}\left(\epsilon_{\nu\}}^{\prime *}(\Delta \epsilon)-\epsilon_{\nu\}}\left(\Delta \epsilon^{\prime *}\right)\right) \mathcal{G}_{5}^{a}(t) \\
& +\frac{1}{2}\left[\Delta_{\{\mu}\left(\epsilon_{\nu\}}^{\prime *}(\Delta \epsilon)+\epsilon_{\nu\}}\left(\Delta \epsilon^{\prime *}\right)\right)\right. \\
& \left.-\epsilon_{\{\mu}^{\prime *} \epsilon_{\nu\}} \Delta^{2}-g_{\mu \nu}\left(\Delta \epsilon^{* *}\right)(\Delta \epsilon)\right] \mathcal{G}_{6}^{a}(t) \\
& +\epsilon_{\{\mu}^{\prime *} \epsilon_{\nu\}} M^{2} \mathcal{G}_{7}^{a}(t)+g_{\mu \nu} M^{2}\left(\epsilon^{\prime *} \epsilon\right) \mathcal{G}_{8}^{a}(t) \\
& +\frac{1}{2} g_{\mu \nu}\left(\Delta \epsilon^{\prime *}\right)(\Delta \epsilon) \mathcal{G}_{9}^{a}(t) \\
& +P_{[\mu}\left(\epsilon_{\nu]}^{\prime *}(\Delta \epsilon)-\epsilon_{\nu]}\left(\Delta \epsilon^{\prime *}\right)\right) \mathcal{G}_{10}^{a}(t) \\
& +\Delta_{[\mu}\left(\epsilon_{\nu]}^{\prime *}(\Delta \epsilon)+\epsilon_{\nu]}\left(\Delta \epsilon^{\prime *}\right)\right) \mathcal{G}_{11}^{a}(t)
\end{aligned}
$$

where $a_{[\mu} b_{v]}=\left(a_{\mu} b_{v}-a_{v} b_{\mu}\right) / 2$. Since one of the two new tensors is non-conserved, energy-momentum conservation imposes the additional sum rule

$$
\sum_{a=q, g} \mathcal{G}_{11}^{a}(t)=0
$$


This is an interesting new feature of the spin- 1 target, since a spin- 0 target has no antisymmetric part and a spin- $1 / 2$ target has only a conserved contribution. This has to do with the fact that the intrinsic generalized angular momentum tensor for a scalar field vanishes, $S_{0}^{\mu \alpha \beta}=0$, and is completely antisymmetric for a Dirac field, as $S_{1 / 2}^{\mu \alpha \beta}=\frac{1}{2} \epsilon^{\mu \alpha \beta \lambda} \bar{\psi} \gamma_{\lambda} \gamma_{5} \psi$ with $\epsilon_{0123}=+1$. In the case of a massive vector field $V^{\mu}$, the intrinsic generalized angular momentum tensor reads $S_{1}^{\mu \alpha \beta}=-2 F^{\mu[\alpha} V^{\beta]}$, so that $\partial_{\alpha} \partial_{\mu} S_{1}^{\mu \alpha \beta} \neq 0$, opening the possibility of having a non-vanishing intrinsic energy dipole moment beside intrinsic angular momentum [48].

Since we will be working with the kinetic form [1] of the QCD EMT, the QCD equations of motion imply that the antisymmetric part of the EMT can be expressed in terms of the axial-vector current as follows $[1,50,51]$

$\left.\bar{\psi} \gamma^{[\mu} i \stackrel{\leftrightarrow}{D^{\nu}}\right] \psi=-\frac{1}{2} \epsilon^{\mu \nu \rho \sigma} \partial_{\rho}\left(\bar{\psi} \gamma_{\sigma} \gamma_{5} \psi\right)$

The matrix elements of the axial-vector current being parametrized as $[52,53]$

$$
\begin{aligned}
\left\langle p^{\prime}, \lambda^{\prime}\left|\bar{\psi}(0) \gamma_{\mu} \gamma_{5} \psi(0)\right| p, \lambda\right\rangle & =-2 i \epsilon_{\mu \alpha \beta P}\left(\epsilon^{\prime * \alpha} \epsilon^{\beta} \tilde{G}_{1}(t)\right. \\
& \left.+\frac{\Delta^{\alpha}\left[\epsilon^{* * \beta}(\Delta \epsilon)-\epsilon^{\beta}\left(\Delta \epsilon^{\prime *}\right)\right]}{M^{2}} \tilde{G}_{2}(t)\right)
\end{aligned}
$$

with the notation $\epsilon_{\mu \alpha \beta P}=\epsilon_{\mu \alpha \beta \lambda} P^{\lambda}$, we find from considering the matrix elements of (7)

$\mathcal{G}_{10}^{q}(t)=-\tilde{G}_{1}(t)+\frac{t}{M^{2}} \tilde{G}_{2}(t)$,

$\mathcal{G}_{11}^{q}(t)=0$,

and $\mathcal{G}_{10}^{g}(t)=\mathcal{G}_{11}^{g}(t)=0$. Note that the vanishing of the antisymmetric part of $T_{\mu \nu}^{g}$ has to do with the impossibility of writing down the gluon spin contribution in a form that is both local and gauge invariant [1]. We thus find that the antisymmetric part of the EMT for a spin-1 hadron is conserved. $^{2}$

\section{Multipole moments of the energy-momentum tensor}

Much of the interesting information about a hadron's mechanical properties that is contained in the EMT is

2 We note that this conclusion holds for the gauge-invariant kinetic EMT, but may not hold for the (non-gauge-invariant) canonical EMT. This is because the gluon contribution to the canonical EMT is asymmetric, and due to the fact that $\partial_{\alpha} \partial_{\mu} S_{1}^{\mu \alpha \beta} \neq 0$, the divergences of the symmetric and antisymmetric parts of the canonical gluon EMT are not expected to separately vanish. encoded by the multipole moments of the EMT matrix elements. These include static observables such as mass, angular momentum, the inertia tensor, and so on, but additionally include information about how each of these decomposes into quark and gluon contributions. The decomposition of hadron mass and angular momentum into quark and gluon contributions - and the latter also into spin and orbital angular momentum components - has been a major focus of recent literature on the EMT. As with much of the other literature on the QCD EMT, this focus has been primarily directed towards spin-1/2 systems (predominantly the proton) and spin-0. In this section, we elaborate on the mechanical properties of spin-1 hadrons encoded by the multipole moments of their EMT, including both properties that are analogous to the lesser-spin cases and those that are new to spin-1.

\subsection{Mass decomposition and balance equation}

The mass decomposition and balance equation associated with a spin-1 target are obtained in terms of the following properly normalized matrix element of the EMT $[5,8]$

$$
\begin{aligned}
\left\langle\iint \mathrm{d}^{3} r T_{\mu \nu}^{a}(0, \boldsymbol{r}) \|\right\rangle & \equiv \frac{\left\langle p, \lambda^{\prime}\left|\int \mathrm{d}^{3} r T_{\mu \nu}^{a}(0, \vec{r})\right| p, \lambda\right\rangle}{\langle p, \lambda \mid p, \lambda\rangle} \\
& =\frac{1}{2 p^{0}}\left\langle p, \lambda^{\prime}\left|T_{\mu \nu}^{a}(0)\right| p, \lambda\right\rangle .
\end{aligned}
$$

Using the covariant expression for the density matrix of a spin-1 system [54,55] (see also App. C for more details)

$\epsilon_{\beta} \epsilon_{\alpha}^{*}=-\frac{1}{3} P_{\beta \alpha}+\frac{i}{2 M} \epsilon_{\beta \alpha \mathcal{S} p}-\mathcal{T}_{\beta \alpha}$,

where the projector onto the subspace orthogonal to $p^{\mu}$ is given by

$P_{\mu \nu}=g_{\mu \nu}-\frac{p_{\mu} p_{v}}{M^{2}}$,

and the covariant vector and tensor polarizations by

$$
\begin{aligned}
& \mathcal{S}^{\mu}(p)=-\epsilon^{\mu \rho \sigma \lambda} \operatorname{Im}\left(\epsilon_{\rho} \epsilon_{\sigma}^{*}\right) \frac{p_{\lambda}}{M}, \\
& \mathcal{T}_{\mu \nu}(p)=-\frac{1}{3} P_{\mu \nu}-\operatorname{Re}\left(\epsilon_{\mu} \epsilon_{\nu}^{*}\right),
\end{aligned}
$$

we find

$$
\begin{aligned}
\left\langle p, \lambda^{\prime}\left|T_{\mu \nu}^{a}(0)\right| p, \lambda\right\rangle= & 2 p_{\mu} p_{\nu}\left[\mathcal{G}_{1}^{a}(0)+\frac{1}{6} \mathcal{G}_{7}^{a}(0)\right] \\
& -2 g_{\mu \nu} M^{2}\left[\frac{1}{2} \mathcal{G}_{8}^{a}(0)+\frac{1}{6} \mathcal{G}_{7}^{a}(0)\right] \\
& -\mathcal{T}_{\mu \nu} M^{2} \mathcal{G}_{7}^{a}(0) .
\end{aligned}
$$

The first two Lorentz structures do not depend on the spin and are indeed common to all targets. The last Lorentz structure is 
new. It is related to the target tensor polarization and therefore does not appear in the case of spin- 0 or spin-1/2 targets. Because of Poincaré invariance, the forward matrix element of the total EMT has to assume the form ${ }^{3}$

$\left\langle p, \lambda^{\prime}\left|T_{\mu \nu}(0)\right| p, \lambda\right\rangle=2 p_{\mu} p_{\nu}$

from which we conclude that

$\sum_{a=q, g} \mathcal{G}_{1}^{a}(0)=1$

using the constraints in Eq. (4).

The Lorentz-invariant coefficients in Eq. (15) can be interpreted in terms of proper internal energy and pressurevolume work [5,8]. In the target rest frame, the partial internal energy is given by 4

$U_{a}=\frac{1}{2 M}\left\langle p, \lambda^{\prime}\left|T_{a}^{00}(0)\right| p, \lambda\right\rangle=\left[\mathcal{G}_{1}^{a}(0)-\frac{1}{2} \mathcal{G}_{8}^{a}(0)\right] M$

and the partial isotropic pressure-volume work by

$W_{a}=\frac{\delta^{i j}}{6 M}\left\langle p, \lambda^{\prime}\left|T_{a}^{i j}(0)\right| p, \lambda\right\rangle=\left[\frac{1}{2} \mathcal{G}_{8}^{a}(0)+\frac{1}{6} \mathcal{G}_{7}^{a}(0)\right] M$.

The new feature of a spin- 1 target is the presence of a partial pressure-volume work anisotropy

$$
\begin{aligned}
W_{a}^{i j} & =\frac{1}{2 M}\left\langle p, \lambda^{\prime}\left|T_{a}^{i j}(0)\right| p, \lambda\right\rangle-\delta^{i j} W_{a} \\
& =\mathcal{T}^{i j}\left[-\frac{1}{2} \mathcal{G}_{7}^{a}(0)\right] M
\end{aligned}
$$

associated with the tensor polarization. The mass decomposition then takes the form

$M=\sum_{a=q, g} U_{a}$

and the balance equations read

$\sum_{a=q, g} W_{a}=0, \quad \sum_{a=q, g} W_{a}^{i j}=0$

3 This is consistent with the total four-mometum of the system being given by $p^{\mu}=\left\langle\left\langle\int \mathrm{d}^{3} r T^{0 \mu}(0, \vec{r})\right\rangle\right\rangle$.

${ }^{4}$ One can of course avoid having recourse to the rest frame and use instead the projectors $p^{\alpha} p^{\beta} / M^{2}$ and $P_{\alpha \beta}$ [5].

\subsection{Angular momentum decomposition}

As explained in detail in $[1,56]$, higher spatial moments of the energy-momentum distribution are ambiguous if defined naively as $\left\langle\left\langle\int \mathrm{d}^{3} r r^{j} T_{\mu \nu}^{a}(0, \vec{r})\right\rangle\right\rangle$. The reason for this is because information about the spatial distribution is lost in the forward limit $\Delta \rightarrow 0$. Spatial distributions can only be defined in frames where no energy is transfered to the system $\Delta^{0}=\vec{P} \cdot \vec{\Delta} / P^{0}=0$. In this work, we will only consider the Breit frame $\vec{P}=\overrightarrow{0}$ where three-dimensional spatial distributions of the EMT are defined as $[4,6,8]$

$\left\langle T_{a}^{\mu \nu}\right\rangle(\vec{r}) \equiv \int \frac{\mathrm{d}^{3} \Delta}{(2 \pi)^{3}} e^{-i \vec{\Delta} \cdot \vec{r}} \frac{1}{2 P^{0}}\left\langle\frac{\vec{\Delta}}{2}, \lambda^{\prime}\left|T_{a}^{\mu \nu}(0)\right|-\frac{\vec{\Delta}}{2}, \lambda\right\rangle$

with $P^{0}=\sqrt{M^{2}+\frac{\vec{\Delta}^{2}}{4}}$. The dipole moment of the spatial distribution is then given by

$$
\begin{aligned}
& \int \mathrm{d}^{3} r r^{j}\left\langle T_{a}^{\mu \nu}\right\rangle(\vec{r}) \\
& =\left\{-i \nabla_{\Delta}^{j}\left[\frac{1}{2 P^{0}}\left\langle\frac{\vec{\Delta}}{2}, \lambda^{\prime}\left|T_{a}^{\mu \nu}(0)\right|-\frac{\vec{\Delta}}{2}, \lambda\right\rangle\right]\right\}_{\Delta=0} .
\end{aligned}
$$

Using the Breit-frame expansion of the polarization fourvector bilinear derived in Appendix C, we find

$$
\begin{aligned}
\int \mathrm{d}^{3} r r^{j}\left\langle T_{a}^{\mu \nu}\right\rangle(\vec{r})= & \frac{1}{2} g^{0\{\mu} \epsilon^{\nu\} j \mathcal{S} 0}\left[\mathcal{G}_{5}^{a}(0)+\frac{1}{2} \mathcal{G}_{7}^{a}(0)\right] \\
& +\frac{1}{2} g^{0[\mu} \epsilon^{\nu] j \mathcal{S} 0} \mathcal{G}_{10}^{a}(0) .
\end{aligned}
$$

Clearly, the only non-vanishing dipole moment is associated with the momentum distribution $\left\langle T_{a}^{0 k}\right\rangle(\vec{r})$ and is orthogonal to the vector polarization of the target $\mathcal{S}^{\mu}=(0, \vec{s})$. It simply originates from the parton orbital angular momentum $(\mathrm{OAM})^{5}$

$$
\begin{aligned}
L_{a}^{i} & =\epsilon^{i j k} \int \mathrm{d}^{3} r r^{j}\left\langle T_{a}^{0 k}\right\rangle(\vec{r}) \\
& =\frac{s^{i}}{2}\left[\mathcal{G}_{5}^{a}(0)+\frac{1}{2} \mathcal{G}_{7}^{a}(0)+\mathcal{G}_{10}^{a}(0)\right] .
\end{aligned}
$$

In QCD, we then find that the parton total angular momentum $(\mathrm{AM})$ is given in the target rest frame by

$$
J_{a}^{i}=\epsilon^{i j k} \int \mathrm{d}^{3} r r^{j}\left\langle T_{a}^{\{0 k\}}\right\rangle(\vec{r})=\frac{s^{i}}{2}\left[\mathcal{G}_{5}^{a}(0)+\frac{1}{2} \mathcal{G}_{7}^{a}(0)\right] .
$$

\footnotetext{
5 Since we consider the local gauge-invariant EMT, we are dealing with the kinetic form of OAM, see e.g. $[1,57]$ for more details.
} 
We naturally recover $J_{q}^{i}=L_{q}^{i}+S_{q}^{i}$ with the quark spin contribution being given by

$S_{q}^{i}=\frac{1}{2} \int \mathrm{d}^{3} r\left\langle\bar{\psi} \gamma^{i} \gamma_{5} \psi\right\rangle(\vec{r})=\frac{s^{i}}{2} \tilde{G}_{1}(0)=-\frac{s^{i}}{2} \mathcal{G}_{10}^{q}(0)$.

For gluons we simply have $J_{g}^{i}=L_{g}^{i}$ because no local gaugeinvariant definition of the gluon spin does exist, see e.g. [1] for a recent detailed discussion.

Beside the term proportional to $\mathcal{G}_{5}^{a}$ already obtained in [37], we also find a contribution from the $\mathcal{G}_{7}^{a}$ GFF describing the non-conserved part of the EMT. Interestingly, such a contribution cannot appear for spin- 0 and $1 / 2$ targets, since in these cases the non-conserved terms are necessarily of the form of a pure trace and hence decoupled from AM. Summing over all the partons, the $\mathcal{G}_{7}$ contribution drops out according to Eq. (4) and we get the AM constraint

$$
\sum_{a=q, g} \mathcal{G}_{5}^{a}(0)=2
$$

first derived in [21].

\subsection{Mass radius and inertia tensor}

Beside mass, two other important quantities characterizing the energy distribution can be defined, namely the mass radius and the inertia tensor. Both are expressed in terms of the following second-order moments

$$
\begin{aligned}
C_{a}^{i j} & =\int \mathrm{d}^{3} r r^{i} r^{j}\left\langle T_{a}^{00}\right\rangle(\vec{r}) \\
& =\left\{-\nabla_{\Delta}^{i} \nabla_{\Delta}^{j}\left[\frac{1}{2 P^{0}}\left\langle\frac{\vec{\Delta}}{2}, \lambda^{\prime}\left|T_{a}^{00}(0)\right|-\frac{\vec{\Delta}}{2}, \lambda\right\rangle\right]\right\}_{\Delta=0} .
\end{aligned}
$$

The mass radius defined as $R_{M}=\sqrt{\left\langle r^{2}\right\rangle}$ with

$\left\langle r^{2}\right\rangle=\frac{1}{M} \sum_{a=q, g} C_{a}^{i j} \delta^{i j}$

gives an idea of the spatial extension of the energy distribution. The inertia tensor $[58,59]$ defined as

$I_{a}^{i j}=C_{a}^{k l}\left(\delta^{k l} \delta^{i j}-\delta^{i k} \delta^{j l}\right)$

allows one to determine the moment of inertia $I_{a}^{\vec{n}}=I_{a}^{i j} n^{i} n^{j}$ of the system about an arbitrary axis $\vec{n}$ passing through the center of mass, which coincides in the Breit frame with the origin [48]. It is related to the mass quadrupole moment
$Q_{a}^{i j}=C_{a}^{k l}\left(\delta^{i k} \delta^{j l}-\frac{1}{3} \delta^{k l} \delta^{i j}\right)=-I_{a}^{k l}\left(\delta^{i k} \delta^{j l}-\frac{1}{3} \delta^{k l} \delta^{i j}\right)$

which measures the deviation from a spherical distribution of the energy.

Using once more the Breit-frame expansion of the polarization four-vector bilinear derived in Appendix C, we find

$$
C_{a}^{i j}=\frac{1}{M}\left[\delta^{i j}\left(\mathcal{A}_{a}(0)+\frac{1}{3} \mathcal{B}_{a}(0)\right)-\mathcal{T}^{i j} \mathcal{B}_{a}(0)\right]
$$

with

$$
\begin{aligned}
\mathcal{A}_{a}(t)= & -\frac{1}{4}\left(\mathcal{G}_{1}^{a}(t)+2 \mathcal{G}_{3}^{a}(t)+\frac{1}{2} \mathcal{G}_{8}^{a}(t)\right) \\
& +2 M^{2} \frac{\mathrm{d}}{\mathrm{d} t}\left(\mathcal{G}_{1}^{a}(t)-\frac{1}{2} \mathcal{G}_{8}^{a}(t)\right), \\
\mathcal{B}_{a}(t)= & -\mathcal{G}_{1}^{a}(t)-\mathcal{G}_{2}^{a}(t)+\mathcal{G}_{5}^{a}(t)+\frac{1}{2} \mathcal{G}_{6}^{a}(t) \\
& +\frac{1}{4} \mathcal{G}_{7}^{a}(t)+\frac{1}{2} \mathcal{G}_{8}^{a}(t)-\frac{1}{2} \mathcal{G}_{9}^{a}(t) .
\end{aligned}
$$

The squared mass radius, inertia tensor and mass quadrupole moment are then given by

$$
\begin{aligned}
\left\langle r^{2}\right\rangle & =\frac{1}{M^{2}} \sum_{a=q, g}\left[3 \mathcal{A}_{a}(0)+\mathcal{B}_{a}(0)\right], \\
I_{a}^{i j} & =\frac{1}{M}\left[2 \delta^{i j}\left(\mathcal{A}_{a}(0)+\frac{1}{3} \mathcal{B}_{a}(0)\right)+\mathcal{T}^{i j} \mathcal{B}_{a}(0)\right], \\
Q_{a}^{i j} & =-\frac{1}{M} \mathcal{T}^{i j} \mathcal{B}_{a}(0) .
\end{aligned}
$$

As expected, the quadrupole moment in spin-1 hadrons is different from zero due to the presence of the tensor polarization.

\section{GFFs as moments of GPDs}

The connection between the EMT and partonic distributions has long been a topic of consideration (see e.g. [60-62]). Generalized parton distributions (GPDs) in particular allow for GFFs to be extracted from their Mellin moments. Since GPDs parametrize the non-perturbative structure contributing to hard reactions such as deeply virtual compton scattering (DVCS) and virtual meson production, they are the most promising avenue for experimentally exploring the form factors appearing in the EMT decomposition.

Leading-twist GPDs have been extensively studied for their polynomiality relations [62]. A specific case allows the second Mellin moments of helicity-independent twist2 GPDs to be related to the non-trace GFFs appearing in the 
symmetric component of the EMT. Such relations have been studied not only for spin-0 [12,63,64] and spin-1/2 [8,61,62], but also spin-1 systems [21,22,37,65].

Beyond leading twist, the Penttinen-Polyakov-ShuvaevStrikman (PPSS) sum rule [66] relates the second moment of a twist-3 GPD to the orbital angular momentum carried by quarks in spin-1/2 hadrons, and it has also been shown [1] that twist-4 GPDs contain information about the nonconserved GFF in a nucleon. In this section we will derive a spin-1 analogue of the PPSS sum rule.

We proceed to derive sum rules for second Mellin moments of GPDs up to twist- $3{ }^{6}$ Consider the quark and gluon vector correlators. ${ }^{7}$

$$
\begin{aligned}
V_{\lambda \lambda^{\prime}}^{\mu, q}= & \left\langle p^{\prime}, \lambda^{\prime}\left|\mathcal{O}_{q V}^{\mu}\right| p, \lambda\right\rangle \\
= & \frac{1}{2} \int_{-\infty}^{\infty} \frac{\mathrm{d} \kappa}{2 \pi} e^{i(P n) \kappa x} \\
& \times\left\langle p^{\prime}, \lambda^{\prime}\left|\bar{\psi}\left(-\frac{n \kappa}{2}\right) \gamma^{\mu}\left[-\frac{n \kappa}{2}, \frac{n \kappa}{2}\right] \psi\left(\frac{n \kappa}{2}\right)\right| p, \lambda\right\rangle,
\end{aligned}
$$

$$
\begin{aligned}
V_{\lambda \lambda^{\prime}}^{\mu, g}= & \left\langle p^{\prime}, \lambda^{\prime}\left|\mathcal{O}_{g V}^{\mu}\right| p, \lambda\right\rangle \\
= & \frac{\delta_{\{\alpha}^{\mu} n_{\beta\}}}{x(P n)} \int_{-\infty}^{\infty} \frac{\mathrm{d} \kappa}{2 \pi} e^{i(P n) \kappa x} \\
& \times\left\langle p^{\prime}, \lambda^{\prime}\right| \operatorname{Tr}\left\{\left[\frac{n \kappa}{2},-\frac{n \kappa}{2}\right]\right. \\
& \left.\times F^{\alpha \lambda}\left(-\frac{n \kappa}{2}\right)\left[-\frac{n \kappa}{2}, \frac{n \kappa}{2}\right] F_{\lambda}^{\beta}\left(\frac{n \kappa}{2}\right)\right\}|p, \lambda\rangle,
\end{aligned}
$$

where $n$ is a light-like four-vector and $[y, z]$ denotes a straight Wilson line joining the spacetime points $y$ and $z$. These correlators enter the description of deeply virtual Compton scattering and can be parametrized up to twist 3 as follows ${ }^{8}[53,68]$

$$
\begin{aligned}
V_{\lambda \lambda^{\prime}}^{\mu, a}= & -\frac{P^{\mu}}{(P n)}\left(\epsilon^{\prime *} \epsilon\right) H_{1}^{a}+\frac{\epsilon^{\prime * \mu}(\Delta \epsilon)-\epsilon^{\mu}\left(\Delta \epsilon^{\prime *}\right)}{2(P n)} H_{2}^{a} \\
& +\frac{P^{\mu}}{(P n)} \frac{\left(\Delta \epsilon^{\prime *}\right)(\Delta \epsilon)}{2 M^{2}} H_{3}^{a}-\frac{\epsilon^{\prime * \mu}(\Delta \epsilon)+\epsilon^{\mu}\left(\Delta \epsilon^{\prime *}\right)}{2(P n)} H_{4}^{a} \\
& +\left[M^{2} \frac{\epsilon^{\prime * \mu}(n \epsilon)+\epsilon^{\mu}\left(n \epsilon^{\prime *}\right)}{2(P n)^{2}}+\frac{1}{3} \frac{P^{\mu}}{(P n)}\left(\epsilon^{\prime *} \epsilon\right)\right] H_{5}^{a} \\
& -\frac{\Delta_{T}^{\mu}}{(P n)}\left(\epsilon^{\prime *} \epsilon\right) G_{1}^{a}-\frac{\Delta_{T}^{\mu}}{(P n)} \frac{\left(n \epsilon^{\prime *}\right)(\Delta \epsilon)-(n \epsilon)\left(\Delta \epsilon^{\prime *}\right)}{2(P n)} G_{2}^{a} \\
& +\frac{\Delta_{T}^{\mu}}{(P n)} \frac{\left(\Delta \epsilon^{\prime *}\right)(\Delta \epsilon)}{2 M^{2}} G_{3}^{a} \\
& -\frac{\Delta_{T}^{\mu}}{(P n)} \frac{\left(n \epsilon^{\prime *}\right)(\Delta \epsilon)+(n \epsilon)\left(\Delta \epsilon^{\prime *}\right)}{2(P n)} G_{4}^{a}
\end{aligned}
$$

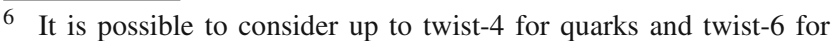
gluons, but contributions beyond twist- 3 are less promising for future phenomenological studies.

7 The Ji convention [62] is used in this work for the gluon GPDs to

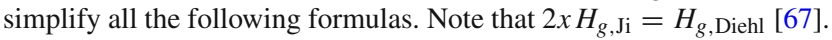

${ }^{8}$ For the complete covariant parametrization, see Appendix D.
}

$$
\begin{aligned}
& +\frac{\Delta_{T}^{\mu}}{(P n)}\left[M^{2} \frac{\left(n \epsilon^{\prime *}\right)(n \epsilon)}{(P n)^{2}}+\frac{1}{3}\left(\epsilon^{\prime *} \epsilon\right)\right] G_{5}^{a} \\
& +\frac{\epsilon_{T}^{\prime * \mu}(\Delta \epsilon)-\epsilon_{T}^{\mu}\left(\Delta \epsilon^{\prime *}\right)}{2(P n)} G_{6}^{a}-\frac{\epsilon_{T}^{\prime * \mu}(\Delta \epsilon)+\epsilon_{T}^{\mu}\left(\Delta \epsilon^{\prime *}\right)}{2(P n)} G_{7}^{a} \\
& +M^{2} \frac{\epsilon_{T}^{\prime * \mu}(n \epsilon)+\epsilon_{T}^{\mu}\left(n \epsilon^{\prime *}\right)}{2(P n)^{2}} G_{8}^{a} \\
& +M^{2} \frac{\epsilon_{T}^{\prime * \mu}(n \epsilon)-\epsilon_{T}^{\mu}\left(n \epsilon^{\prime *}\right)}{2(P n)^{2}} G_{9}^{a} .
\end{aligned}
$$

The first five terms $\left(H_{i}^{a}\right)$ correspond to the twist-2 GPDs, and the remaining nine $\left(G_{i}^{a}\right)$ are purely twist-3. In the quark sector, the twist-3 GPDs satisfy the relation $\int \mathrm{d} x G_{i}^{q}=0$ as a consequence of the charge current conservation. We suppressed the dependence of the GPDs on the parton longitudinal momentum $x$, longitudinal momentum transfer $\xi=-(\Delta n) / 2(P n)$, and squared momentum transfer $t=\Delta^{2}$ for conciseness of notation, and made use of the Sudakov decomposition of four-vectors $\left(n^{2}=\bar{n}^{2}=0, n \bar{n}=1\right)$

$z^{\mu}=(z n) \bar{n}^{\mu}+(z \bar{n}) n^{\mu}+z_{T}^{\mu}$

The second Mellin moment of the light-front string operators are related to the EMT up to twist 3 as follows

$\int_{-1}^{1} \mathrm{~d} x x \mathcal{O}_{q V}^{\mu}=\frac{1}{4(P n)^{2}} \bar{\psi}(0) \gamma^{\mu}(i \stackrel{\leftrightarrow}{D} n) \psi(0)=\frac{T_{q}^{\mu n}}{2(P n)^{2}}$,

$\int_{-1}^{1} \mathrm{~d} x \times \mathcal{O}_{g V}^{\mu}=\frac{1}{(P n)^{2}} \operatorname{Tr}\left[F^{\mu \lambda}(0) F_{\lambda n}(0)\right]=\frac{T_{g}^{\mu n}}{2(P n)^{2}}$.

Taking the off-forward matrix element on both sides allows us to relate seven of the GFFs to moments of leading-twist vector GPDs [37,65]. Comparing the Mellin moment of Eq. (40) with the decomposition in Eq. (2) in the symmetric frame $P_{T}^{\mu}=0$, we find the following relations for quarks and gluons at twist 2

$$
\int_{-1}^{1} \mathrm{~d} x x\left[H_{1}^{a}(x, \xi, t)-\frac{1}{3} H_{5}^{a}(x, \xi, t)\right]=\mathcal{G}_{1}^{a}(t)+\xi^{2} \mathcal{G}_{3}^{a}(t),
$$

$\int_{-1}^{1} \mathrm{~d} x \times H_{2}^{a}(x, \xi, t)=\mathcal{G}_{5}^{a}(t)$,

$\int_{-1}^{1} \mathrm{~d} x \times H_{3}^{a}(x, \xi, t)=\mathcal{G}_{2}^{a}(t)+\xi^{2} \mathcal{G}_{4}^{a}(t)$,

$\int_{-1}^{1} \mathrm{~d} x \times H_{4}^{a}(x, \xi, t)=\xi \mathcal{G}_{6}^{a}(t)$,

$\int_{-1}^{1} \mathrm{~d} x \times H_{5}^{a}(x, \xi, t)=-\frac{t}{4 M^{2}} \mathcal{G}_{6}^{a}(t)+\frac{1}{2} \mathcal{G}_{7}^{a}(t)$.

Since the GFFs appear as second Mellin moments of GPDs, they are special cases of generalized form factors, which cor- 
respond to arbitrary moments of GPDs. We note the following correspondence between the GFFs as defined in this work, and the $s=2$ generalized form factors appearing in Ref. [65]:

$A_{2,0}^{a}(t)=\mathcal{G}_{1}^{a}(t)$,

$B_{2,0}^{a}(t)=\mathcal{G}_{5}^{a}(t)$,

$C_{2,0}^{a}(t)=\mathcal{G}_{2}^{a}(t)$

$2 D_{2,1}^{a}(t)=-\mathcal{G}_{6}^{a}(t)$,

$E_{2,1}^{a}(t)=-\frac{t}{4 M^{2}} \mathcal{G}_{6}^{a}(t)+\frac{1}{2} \mathcal{G}_{7}^{a}(t)$,

$4 F_{2}^{a}(t)=\mathcal{G}_{3}^{a}(t)$,

$4 H_{2}^{a}(t)=\mathcal{G}_{4}^{a}(t)$.

We also find the following relations for quarks at twist 3

$$
\begin{aligned}
& \int_{-1}^{1} \mathrm{~d} x x G_{1}^{q}(x, \xi, t)=-\frac{\xi}{2} \mathcal{G}_{3}^{q}(t) \\
& =-\frac{1}{4} \frac{\partial}{\partial \xi} \int_{-1}^{1} \mathrm{~d} x x\left[H_{1}^{q}(x, \xi, t)-\frac{1}{3} H_{5}^{q}(x, \xi, t)\right],
\end{aligned}
$$$$
\int_{-1}^{1} \mathrm{~d} x x G_{2}^{q}(x, \xi, t)=0,
$$$$
\int_{-1}^{1} \mathrm{~d} x x G_{3}^{q}(x, \xi, t)=-\frac{\xi}{2} \mathcal{G}_{4}^{q}(t)
$$$$
=-\frac{1}{4} \frac{\partial}{\partial \xi} \int_{-1}^{1} \mathrm{~d} x x H_{3}^{q}(x, \xi, t),
$$$$
\int_{-1}^{1} \mathrm{~d} x x G_{4}^{q}(x, \xi, t)=-\frac{1}{4} \mathcal{G}_{6}^{q}(t)
$$$$
=-\frac{1}{4} \frac{\partial}{\partial \xi} \int_{-1}^{1} \mathrm{~d} x x H_{4}^{q}(x, \xi, t),
$$$$
\int_{-1}^{1} \mathrm{~d} x x G_{5}^{q}(x, \xi, t)=0,
$$$$
\int_{-1}^{1} \mathrm{~d} x x G_{6}^{q}(x, \xi, t)=-\frac{1}{2}\left[\mathcal{G}_{5}^{q}(t)+\mathcal{G}_{10}^{q}(t)\right]
$$$$
=-\frac{1}{2} \int_{-1}^{1} \mathrm{~d} x \times H_{2}^{q}(x, \xi, t)+\frac{1}{2}\left[\tilde{G}_{1}(t)-\frac{t}{M^{2}} \tilde{G}_{2}(t)\right],
$$$$
\int_{-1}^{1} \mathrm{~d} x x G_{7}^{q}(x, \xi, t)=-\frac{\xi}{2} \mathcal{G}_{6}^{q}(t)
$$$$
=-\frac{1}{2} \int_{-1}^{1} \mathrm{~d} x x H_{4}^{q}(x, \xi, t),
$$$$
\int_{-1}^{1} \mathrm{~d} x x G_{8}^{q}(x, \xi, t)=0,
$$$$
\int_{-1}^{1} \mathrm{~d} x x G_{9}^{q}(x, \xi, t)=0,
$$

where we have used Eq. (9b).
Based on Eq. (27), we find that the total quark or gluon $\mathrm{AM}$ in a state with maximal vector polarization along the $z$-direction can be expressed in terms of twist-2 GPDs as follows

$J_{a}^{z}=\int_{-1}^{1} \mathrm{~d} x \frac{x}{2}\left[H_{2}^{a}(x, 0,0)+H_{5}^{a}(x, 0,0)\right]$,

which is nothing but Ji's relation [61] for spin-1 targets. Summing over quark and gluon contributions, we recover the spin sum rule derived in [21]

$J^{z}=\sum_{a=q, g} \int_{-1}^{1} \mathrm{~d} x \frac{x}{2} H_{2}^{a}(x, 0,0)=1$.

Unlike the case of spin- $1 / 2$ targets $[66,69,70]$, the quark OAM (26) requires not only a pure twist-3 GPD but also a twist-2 GPD

$L_{q}^{z}=\int_{-1}^{1} \mathrm{~d} x x\left[\frac{1}{2} H_{5}^{q}(x, 0,0)-G_{6}^{q}(x, 0,0)\right]$.

This twist-2 GPD contribution is associated with the tensor polarization and is therefore absent in the case of spin- $1 / 2$ targets.

Quark and gluon contributions to mass and pressurevolume work involve trace terms and hence twist-4 GPDs. Only the partial pressure-volume work anisotropy can be related to a twist-2 GPD

$W_{a}^{i j}=-\mathcal{T}^{i j} M \int_{-1}^{1} \mathrm{~d} x x H_{5}^{a}(x, 0,0)$.

Summing over quarks and gluons, the mass sum rule and the balance equations imply the following constraints

$$
\begin{aligned}
& \sum_{a=q, g} \int_{-1}^{1} \mathrm{~d} x \times H_{1}^{a}(x, 0,0)=1, \\
& \sum_{a=q, g} \int_{-1}^{1} \mathrm{~d} x x H_{5}^{a}(x, 0,0)=0 .
\end{aligned}
$$

These relations can be interpreted as statements of energymomentum conservation for collinear parton distribution functions (PDFs). Using the notation of $[31,71],{ }^{9}$ the unpolarized and tensor-polarized PDFs are given respectively by $f_{1}^{a}(x)=H_{1}^{a}(x, 0,0)$ and $f_{1 L L}^{a}(x)=H_{5}^{a}(x, 0,0)$. In the case of quarks, they enter the deep inelastic structure functions $F_{1}$ and $b_{1}$ at leading order and leading twist as

\footnotetext{
${ }^{9}$ Note that Ref. [31] uses $(P n)=1$.
} 


$$
\begin{aligned}
F_{1}\left(x, Q^{2}\right)= & \frac{1}{2} \sum_{q} e_{q}^{2}\left[H_{1}^{q}\left(x, 0,0 ; \mu^{2}=Q^{2}\right)\right. \\
& \left.-H_{1}^{q}\left(-x, 0,0 ; \mu^{2}=Q^{2}\right)\right], \\
b_{1}\left(x, Q^{2}\right)= & \frac{1}{2} \sum_{q} e_{q}^{2}\left[H_{5}^{q}\left(x, 0,0 ; \mu^{2}=Q^{2}\right)\right. \\
& \left.-H_{5}^{q}\left(-x, 0,0 ; \mu^{2}=Q^{2}\right)\right] .
\end{aligned}
$$

The corresponding gluon PDFs mix with the quark ones and contribute to the DIS structure functions at higher order in the $\alpha_{s}$ perturbative expansion. Thus Eq. (50) is a statement of the momentum sum rule for PDFs. The collinear structure functions for a scattering off a tensor polarized targets were first introduced in $[20,25,72]$ and the separate contributions of quarks and gluons to Eq. (51) were previously discussed in $[73,74]$.

\section{Conclusion}

In this work, we found the most general form that the asymmetric, gauge-invariant kinetic energy-momentum tensor (EMT) of a spin-1 hadron can take. Expressions were given for both the full EMT and the partial EMT due to a single parton type. We explored the physical meaning of the gravitational form factors appearing in this EMT, including sum rules imposed by conservation of momentum and angular momentum, the decomposition of spin-1 hadron mass, and multipole moments of the EMT. We also explored connections between the gravitational form factors and other functions describing partonic structure, such as axial form factors and generalized parton distributions up to twist three.

The spin-1 EMT was found to contain many more gravitational form factors than the corresponding spin-0 or spin- $1 / 2$ EMTs. A total of 11 form factors are present in the EMT decomposition, with 9 of these in the symmetric part and 2 in the antisymmetric part. Among them, 6 structures have no analogues in the lower-spin cases and are related to the presence of tensor polarization modes. They contribute to features new to spin-1 hadrons such as a quadrupole moment and possibly a non-zero intrinsic energy dipole moment aside from the intrinsic angular momentum.

The structure of the spin-1 EMT is rich, and there remains much to be explored. The pressure and shear force distributions encoded within it, and how these differ from the simpler spin- 0 case, are worthy of detailed study. It is also worth investigating how the EMT of a composite spin-1 hadron compares to that of an elementary spin-1 particle, such as a photon or one of the heavy electroweak gauge bosons. These topics will be the subject of future work, along with illustrative model calculations.
Experimentally, measurements of coherent hard exclusive processes with deuteron targets are possible at JLab and the future EIC with forward detectors. Extraction of the chiral-even vector GPDs from these measurements would then constrain the deuteron gravitational form factors through the Mellin moments of these GPDs. Similarly, extraction of GDAs for the rho-rho meson pair from the crossed reaction $\gamma^{*} \gamma \rightarrow \rho \rho$ at Belle would constrain the rho meson gravitational form factors.

Note

Shortly after the present work was completed and made available, the independent work [75] appeared, dealing with the EMT of spin-1 hadrons. The results are consistent with ours and the document contains an especially useful comparison between the different nomenclature for the GFFs present in the literature.

Acknowledgements We are grateful to Bernard Pire and Maxim Polyakov for useful discussions. The work of SC and CL was supported by the Agence Nationale de la Recherche under the projects No. ANR-18-ERC1-0002 and ANR-16-CE31-0019. AF was supported by the U.S. Department of Energy, Office of Science, Office of Nuclear Physics, Contract No. DE-AC02-06CH11357 and an LDRD initiative at Argonne National Laboratory under Project No. 2017-058-N0.

Data Availability Statement This manuscript has no associated data or the data will not be deposited. [Authors' comment: Data sharing not applicable to this article as no data sets were generated or analyzed during the current study.]

Open Access This article is distributed under the terms of the Creative Commons Attribution 4.0 International License (http://creativecomm ons.org/licenses/by/4.0/), which permits unrestricted use, distribution, and reproduction in any medium, provided you give appropriate credit to the original author(s) and the source, provide a link to the Creative Commons license, and indicate if changes were made.

Funded by $\mathrm{SCOAP}^{3}$.

\section{Appendix A: Form factor counting}

Let us consider the total number of GFFs we may expect to appear in the decomposition of the spin-1 EMT. This will depend on the EMT definition we use. First, let us consider the most general possible rank-2 tensor that is even under charge conjugation. It decomposes into several representations of the Lorentz group, namely $(0,0),(1,0) \oplus(0,1)$, and $(1,1)$, which are respectively 1-, 6-, and 9-dimensional representations. These representations have $J^{P C}$ quantum number decompositions given in Table 1 .

With these $J^{P C}$ numbers, we can use the method of Ji \& Lebed to count form factors [76].

We consider a crossed channel matrix element $\langle h \bar{h}|\hat{\mathcal{O}}| 0\rangle$, and write out all the possible $J^{P C}(L)$ quantum numbers that $h \bar{h}$ can have up to $J=2$. We then count the matches between 
Table $1 J^{P C}$ quantum numbers of several representations of the Lorentz group, constrained to be $\mathrm{C}$-even

\begin{tabular}{ll}
\hline Rep. & $J^{P C}$ \\
\hline$(0,0)$ & $0^{++}$ \\
$(1,0) \oplus(0,1)$ & $1^{++}, 1^{-+}$ \\
$(1,1)$ & $0^{++}, 1^{-+}, 2^{++}$ \\
\hline
\end{tabular}

Table 2 Limited list of $J_{L}^{P C}(S)$ quantum numbers possible for a $h \bar{h}$ state. Only $J^{P C}$ numbers present in Table 1 are included here. The lists for spin- 0 and spin- 1 are from $[65,77,78]$, while the spin- $1 / 2$ list is from [76]

\begin{tabular}{ll}
\hline Spin & $J_{L}^{P C}(S)$ \\
\hline 0 & $0_{0}^{++}(0), 2_{2}^{++}(0)$ \\
$\frac{1}{2}$ & $0_{1}^{++}(1), 1_{1}^{++}(1), 2_{1}^{++}(1), 2_{3}^{++}(1)$ \\
1 & $0_{0}^{++}(0), 0_{2}^{++}(2), 1_{2}^{++}(2), 1_{1}^{-+}(1), 2_{2}^{++}(0), 2_{0,2,4}^{++}(2)$ \\
\hline
\end{tabular}

Table 3 The number of form factors in a decomposition of a rank-2 tensor belonging to any of the representations listed in the table

\begin{tabular}{llll}
\hline Rep. & Spin-0 GFFs & Spin- $\frac{1}{2}$ GFFs & Spin-1 GFFs \\
\hline$(0,0)$ & 1 & 1 & 2 \\
$(1,0) \oplus(0,1)$ & 0 & 1 & 2 \\
$(1,1)$ & 2 & 3 & 7 \\
\hline
\end{tabular}

these $J^{P C}$ and those in our hypothetical rank-2 tensor. Such lists can be found in Table 2 .

With these lists in hand, we can count the number of form factors that are contributing to the general rank-2 tensor by each of the representations it decomposes into. The numbers are given in Table 3.

Each of the rows in Table 3 tells us something meaningful about form factor counts.

- The first row gives us trace terms, which can be thought of as "non-conserving" terms. There is one such term for both spin- 0 and spin-1/2, and they are associated with the $\bar{C}(t)$ GFFs. For spin-1, there are two non-conserving terms of this kind.

- The second row tells us the number of GFFs appearing in the antisymmetric part of the EMT.

- The third row tells us the number of GFFs in the decomposition of a traceless symmetric rank-2 tensor, and they correspond to the number of GFFs that are known to appear in the second Mellin moments of twist-2 vector GPDs.

\section{Appendix B: Parametrization with Lorentz projectors}

The matrix elements of the most general local, gaugeinvariant EMT for a massive spin-1 target can be written as

$\left\langle p^{\prime}, \lambda^{\prime}\left|T_{\mu \nu}(0)\right| p, \lambda\right\rangle=\epsilon^{* \alpha}\left(p^{\prime}, \lambda^{\prime}\right) T_{\mu \nu, \alpha \beta}(P, \Delta) \epsilon^{\beta}(p, \lambda)$,

where the effective vertex $T_{\mu v, \alpha \beta}(P, \Delta)$ is a Lorentz tensor constructed out of the invariant tensors $g_{\mu \nu}$ and $\epsilon_{\mu \nu \alpha \beta}$, and the available four-vectors $P$ and $\Delta$. Because of the onshell relations $\left(p^{\prime} \epsilon^{\prime *}\right)=(p \epsilon)=0$, we choose to discard terms involving $P_{\alpha}$ or $P_{\beta}$ as they are not independent from those involving $\Delta_{\alpha}$ or $\Delta_{\beta}$. Finally, discrete symmetries impose further constraints on the effective vertex. Invariance under parity and time reversal implies that

$T_{\mu \nu, \alpha \beta}(P, \Delta)=T_{\bar{\mu} \bar{\nu}, \bar{\alpha} \bar{\beta}}(\bar{P}, \bar{\Delta})=T_{\bar{\mu} \bar{\nu}, \bar{\alpha} \bar{\beta}}^{*}(\bar{P}, \bar{\Delta})$,

where a bar over a four-vector or a Lorentz index stands for the parity-transformed object $\bar{a}^{\mu}=a^{\bar{\mu}}=\left(a^{0},-\boldsymbol{a}\right)$. The hermiticity property leads to the last constraint

$T_{\mu \nu, \alpha \beta}(P, \Delta)=T_{\mu \nu, \beta \alpha}^{*}(P,-\Delta)$.

It follows from these constraints that the effective vertex is real, and that its symmetric part under the exchange $\alpha \leftrightarrow \beta$ involves only even powers of $\Delta$, whereas the antisymmetric part involves only odd powers of $\Delta$. Note also that the Levi-Civita tensor need not be considered since it is the only object with negative intrinsic parity and since the product $\epsilon_{\mu \nu \alpha \beta} \epsilon_{\rho \sigma \tau \lambda}$ can be rewritten in terms of the metric only.

Interestingly, we can find a complete parametrization of the effective vertex in terms of the Lorentz projectors onto $(0,0),(1,0) \oplus(0,1)$, and $(1,1)$ representations

$$
\begin{aligned}
& \left(I_{\mu \nu}\right)_{\alpha \beta}=\frac{1}{4} g_{\mu \nu} g_{\alpha \beta}, \\
& \left(D_{\mu \nu}\right)_{\alpha \beta}=\frac{1}{2}\left(g_{\mu \alpha} g_{\nu \beta}-g_{\mu \beta} g_{\nu \alpha}\right), \\
& \left(Q_{\mu \nu}\right)_{\alpha \beta}=\frac{1}{2}\left(g_{\mu \alpha} g_{\nu \beta}+g_{\mu \beta} g_{\nu \alpha}\right)-\frac{1}{4} g_{\mu \nu} g_{\alpha \beta} .
\end{aligned}
$$

We recognize in particular the generators of Lorentz transformations $\left(M^{\mu \nu}\right)^{\alpha \beta}=2 i\left(D^{\mu \nu}\right)^{\alpha \beta}$ associated with the vector representation $\left(\frac{1}{2}, \frac{1}{2}\right)$. Taking into account all the constraints discussed above, we find that the most general effective vertex can be written as a linear combination of the following eleven independent Lorentz structures (omitting the $\alpha, \beta$ indices for convenience)

$I_{\mu \nu}, P_{\mu} I_{\nu P}, \Delta_{\mu} I_{\nu \Delta}$, $P_{\mu} D_{\nu \Delta}, P_{\nu} D_{\mu \Delta}$, 
$Q_{\mu \nu}, \Delta_{\mu} Q_{\nu \Delta}, \Delta_{\nu} Q_{\mu \Delta}, g_{\mu \nu} Q_{\Delta \Delta}, P_{\mu} P_{\nu} Q_{\Delta \Delta}, \Delta_{\mu} \Delta_{\nu} Q_{\Delta \Delta}$.

At first sight, it seems that these are at odds with the counting in Appendix A, since we found three structures involving the projector onto the $(0,0)$ representation and six involving the projector onto the $(1,1)$ representation. In fact, the counting in Appendix A corresponds to the projections applied on the pair of indices $\mu \nu$. The structures corresponding to the $(0,0)$ part of the effective vertex are then the only two pure trace terms $I_{\mu \nu}$ and $g_{\mu \nu} Q_{\Delta \Delta}$. The structures corresponding to the $(1,1)$ part of the effective vertex are those obtained from removing the trace of the seven other symmetric terms $P_{\mu} I_{\nu P}, \Delta_{\mu} I_{\nu \Delta}, Q_{\mu \nu}, \Delta_{\mu} Q_{\nu \Delta}, \Delta_{\nu} Q_{\mu \Delta}, P_{\mu} P_{\nu} Q_{\Delta \Delta}$, and $\Delta_{\mu} \Delta_{\nu} Q_{\Delta \Delta}$.

This technique has the advantage of showing the connection with lower spin targets. For spin-0 targets we have $I_{\mu \nu}=\frac{1}{4} g_{\mu \nu}, D_{\mu \nu}=0$ and $Q_{\mu \nu}=0$, and for spin- $1 / 2$ targets we have $I_{\mu \nu}=\frac{1}{4} g_{\mu \nu} \mathbb{I}, D_{\mu \nu}=i \sigma_{\mu \nu}$ and $Q_{\mu \nu}=0 .{ }^{10}$ According to (B5) this leads respectively to 3 and 5 GFFs, in agreement with Appendix A.

\section{Appendix C: Polarization four-vector bilinears}

Matrix elements of spin-1 targets can be written in terms of polarization four-vector bilinears contracted with some Lorentz tensors

$$
\begin{aligned}
& \left\langle p^{\prime}, \lambda^{\prime}\left|O^{\mu_{1} \cdots \mu_{n}}(0)\right| p, \lambda\right\rangle \\
& \quad=\epsilon_{\alpha}^{*}\left(p^{\prime}, \lambda^{\prime}\right) O^{\mu_{1} \cdots \mu_{n}, \alpha \beta}(P, \Delta) \epsilon_{\beta}(p, \lambda) .
\end{aligned}
$$

In the forward limit, the polarization four-vector bilinear reduces to the covariant density matrix which can be written as [54,55]

$$
\begin{aligned}
& \epsilon_{\beta}(p, \lambda) \epsilon_{\alpha}^{*}\left(p, \lambda^{\prime}\right)=\rho_{\beta \alpha}(p) \\
& \quad=\left[\frac{1}{3} \mathbb{I}+\frac{1}{2} \mathcal{S}^{\mu}(p) \Sigma_{\mu}+\mathcal{T}^{\mu \nu}(p) \Sigma_{\mu \nu}\right]_{\beta \alpha},
\end{aligned}
$$

where the vector and tensor polarization operators read

$$
\begin{aligned}
& \Sigma_{\mu}=\frac{1}{2 M} \epsilon_{\mu \rho \sigma p} M^{\rho \sigma}, \\
& \Sigma_{\mu \nu}=-\Sigma_{\{\mu} \Sigma_{\nu\}}+\frac{1}{3}\left(g_{\mu \nu}-\frac{p_{\mu} p_{v}}{M^{2}}\right) \Sigma \cdot \Sigma,
\end{aligned}
$$

and are orthogonal to $p^{\mu}$ as required by the onshell relation $(p \epsilon)=0$. Clearly, the covariant vector and tensor polarizations can be taken so as to satisfy the same properties as the associated operators

\footnotetext{
$\overline{10}$ Interestingly, since $\sigma^{\mu \nu}=\frac{i}{2}\left[\gamma^{\mu}, \gamma^{\nu}\right]$ we can interpret the Clifford algebra $\left\{\gamma^{\mu}, \gamma^{\nu}\right\}=2 g^{\mu \nu} \mathbb{I}$ as the condition of vanishing quadrupole.
}

$p_{\mu} \mathcal{S}^{\mu}(p)=0$,

$\mathcal{T}^{\nu \mu}(p)=\mathcal{T}^{\mu \nu}(p)$,

$p_{\mu} \mathcal{T}^{\mu \nu}(p)=0$,

$g_{\mu \nu} \mathcal{T}^{\mu \nu}(p)=0$.

They carry the dependence on the polarizations $\lambda$ and $\lambda^{\prime}$, which has been omitted for convenience.

The generators of Lorentz transformations in the $\left(\frac{1}{2}, \frac{1}{2}\right)$ representation being given by

$\left(M^{\rho \sigma}\right)_{\alpha \beta}=i\left(\delta_{\alpha}^{\rho} \delta_{\beta}^{\sigma}-\delta_{\beta}^{\rho} \delta_{\alpha}^{\sigma}\right)$,

the unit, vector and tensor polarization operators take the explicit form

$(\mathbb{I})_{\beta \alpha}=-P_{\beta \alpha}$,
$\left(\Sigma_{\mu}\right)_{\beta \alpha}=\frac{i}{M} \epsilon_{\mu \beta \alpha p}$,
$\left(\Sigma_{\mu \nu}\right)_{\beta \alpha}=-P_{\mu\{\beta} P_{\alpha\} \nu}+\frac{1}{3} P_{\mu \nu} P_{\beta \alpha}$,

where $P_{\mu \nu}$ is the projector onto the subspace orthogonal to $p^{\mu}$ given by

$P_{\mu \nu}=g_{\mu \nu}-\frac{p_{\mu} p_{\nu}}{M^{2}}$.

All these operators are orthogonal to each other, so that the normalization, covariant vector and tensor polarizations can simply be obtained as follows

$\operatorname{Tr}[\rho(p) \mathbb{I}]=1$,

$\operatorname{Tr}\left[\rho(p) \Sigma^{\mu}\right]=-\mathcal{S}^{\mu}(p)$,

$\operatorname{Tr}\left[\rho(p) \Sigma^{\mu \nu}\right]=\mathcal{T}^{\mu \nu}(p)$.

Denoting the rest-frame four-momentum as $k^{\mu}=M g^{\mu 0}$, the general polarization four-vector bilinear can be written as

$\epsilon^{\beta}(p, \lambda) \epsilon^{* \alpha}\left(p^{\prime}, \lambda^{\prime}\right)=\left(\Lambda_{\text {can }}\right)_{\rho}^{\beta}\left(\Lambda_{\text {can }}^{\prime}\right)_{\sigma}^{\alpha} \rho^{\rho \sigma}(k)$,

where the standard canonical rotationless Lorentz boost tensor reads

$$
\begin{aligned}
& \left(\Lambda_{\text {can }}\right)^{\mu}{ }_{\nu} \\
& \quad=\left(\begin{array}{cccc}
\frac{p^{0}}{M} & \frac{p^{1}}{M} & \frac{p^{2}}{M} & \frac{p^{3}}{M} \\
\frac{p^{1}}{M} & 1+\frac{\left(p^{1}\right)^{2}}{M\left(p^{0}+M\right)} & \frac{p^{1} p^{2}}{M\left(p^{0}+M\right)} & \frac{p^{1} p^{3}}{M\left(p^{0}+M\right)} \\
\frac{p^{2}}{M} & \frac{p^{1} p^{2}}{M\left(p^{0}+M\right)} & 1+\frac{\left(p^{2}\right)^{2}}{M\left(p^{0}+M\right)} & \frac{p^{2} p^{3}}{M\left(p^{0}+M\right)} \\
\frac{p^{3}}{M} & \frac{p^{1} p^{3}}{M\left(p^{0}+M\right)} & \frac{p^{2} p^{3}}{M\left(p^{0}+M\right)} & 1+\frac{\left(p^{3}\right)^{2}}{M\left(p^{0}+M\right)},
\end{array}\right)
\end{aligned}
$$


or in a more covariant form $[79,80]$

$\left(\Lambda_{\mathrm{can}}\right)_{v}^{\mu}=\delta_{v}^{\mu}-\frac{(p+k)^{\mu}(p+k)_{v}}{k \cdot(p+k)}+\frac{2 p^{\mu} k_{v}}{M^{2}}$.

Denoting for convenience the rest-frame covariant vector and tensor polarizations as

$\mathcal{S}^{\mu} \equiv \mathcal{S}^{\mu}(k), \quad \mathcal{T}^{\mu \nu} \equiv \mathcal{T}^{\mu \nu}(k)$,

we find that the general polarization four-vector bilinear can be expressed as

$$
\begin{aligned}
& \epsilon^{\beta}(p, \lambda) \epsilon^{* \alpha}\left(p^{\prime}, \lambda^{\prime}\right) \\
& =\frac{1}{3}\left[-g^{\beta \alpha}+\frac{p^{\beta} p^{\prime \alpha}}{M^{2}}-\frac{(p+k)^{\beta} \Delta^{\alpha}}{k \cdot(p+k)}\right. \\
& \left.\quad+\frac{\Delta^{\beta}\left(p^{\prime}+k\right)^{\alpha}}{k \cdot\left(p^{\prime}+k\right)}+\frac{\Delta^{2}}{2} \frac{(p+k)^{\beta}\left(p^{\prime}+k\right)^{\alpha}}{[k \cdot(p+k)]\left[k \cdot\left(p^{\prime}+k\right)\right]}\right] \\
& \quad+\frac{i}{2 M}\left[\epsilon^{\beta \alpha \mathcal{S} k}-\epsilon^{\beta p^{\prime} \mathcal{S} k} \frac{\left(p^{\prime}+k\right)^{\alpha}}{k \cdot\left(p^{\prime}+k\right)}\right. \\
& \left.\quad-\frac{(p+k)^{\beta}}{k \cdot(p+k)} \epsilon^{p \alpha \mathcal{S} k}+\epsilon^{p p^{\prime} \mathcal{S} k} \frac{(p+k)^{\beta}\left(p^{\prime}+k\right)^{\alpha}}{[k \cdot(p+k)]\left[k \cdot\left(p^{\prime}+k\right)\right]}\right] \\
& \quad-\mathcal{T}^{\beta \alpha}+\mathcal{T}^{\beta p^{\prime}} \frac{\left(p^{\prime}+k\right)^{\alpha}}{k \cdot\left(p^{\prime}+k\right)}+\frac{(p+k)^{\beta}}{k \cdot(p+k)} \mathcal{T}^{p \alpha} \\
& \quad-\mathcal{T}^{p p^{\prime}} \frac{(p+k)^{\beta}\left(p^{\prime}+k\right)^{\alpha}}{[k \cdot(p+k)]\left[k \cdot\left(p^{\prime}+k\right)\right]} .
\end{aligned}
$$

In the forward limit, we recover the standard expression for the covariant density matrix

$\rho_{\beta \alpha}(p)=-\frac{1}{3} P_{\beta \alpha}+\frac{i}{2 M} \epsilon_{\beta \alpha \rho \sigma} \mathcal{S}^{\rho}(p) p^{\sigma}-\mathcal{T}_{\beta \alpha}(p)$,

where

$\mathcal{S}^{\mu}(p)=\left(\Lambda_{\text {can }}\right)^{\mu}{ }_{\rho} \mathcal{S}^{\rho}, \quad \mathcal{T}^{\mu \nu}(p)=\left(\Lambda_{\text {can }}\right)^{\mu}{ }_{\rho}\left(\Lambda_{\text {can }}\right)^{\nu}{ }_{\sigma} \mathcal{T}^{\rho \sigma}$.

As noticed in the case of Dirac bilinears [81], in the forward limit $\Delta=0$ the dependence on the rest-frame fourmomentum $k^{\mu}$ can be absorbed into the $p$-dependent covariant polarization. However, in the general off-forward case $\Delta \neq 0$ this is not possible anymore and the $k$-dependence remains explicit. This dependence is unavoidable in a relativistic theory and comes from the fact that, because of the non-commutativity of boosts, canonical polarization of a massive particle has to be defined from a boost of the restframe polarization to the frame of interest [82].

In the Breit frame $\vec{P}=\overrightarrow{0}$, we find that the general polarization four-vector bilinear reduces to

$$
\begin{aligned}
\epsilon^{\beta}(p, \lambda) \epsilon^{* \alpha}\left(p^{\prime}, \lambda^{\prime}\right) \\
=\frac{1}{3}\left[-g^{\beta \alpha}+\left(1+\frac{\Delta^{2}}{4 M^{2}}\right) \frac{k^{\beta} k^{\alpha}}{M^{2}}-\frac{k^{[\beta} \Delta^{\alpha]}}{M^{2}}+\frac{\Delta^{\beta} \Delta^{\alpha}}{4 M^{2}}\right] \\
\quad+\frac{i}{2 M}\left[\epsilon^{\beta \alpha \mathcal{S} k}-\frac{k^{\{\beta} \epsilon^{\alpha\} \Delta \mathcal{S} k}}{M^{2}}+\frac{\Delta^{[\beta} \epsilon^{\alpha] \Delta \mathcal{S} k}}{4 M^{2}}\right] \\
\quad-\mathcal{T}^{\beta \alpha}-\frac{k^{[\beta} \mathcal{T}^{\alpha] \Delta}}{M^{2}}+\frac{\Delta^{\{\beta} \mathcal{T}^{\alpha\}} \Delta}{4 M^{2}}+\frac{\mathcal{T}^{\Delta \Delta}}{4 M^{2}} \frac{k^{\beta} k^{\alpha}}{M^{2}}+\mathcal{O}\left(\Delta^{3}\right) .
\end{aligned}
$$

\section{Appendix D: Covariant parametrization of Generalized Parton Distributions}

Just like for local operators, hadronic matrix elements of non-local operators can be parametrized in a covariant form if one includes in the list of available four-vectors the direction of non-locality, namely the lightlike four-vector $n$ in the case of parton distributions like GPDs. The most general parametrization of the quark GPD correlator (39a), that respects the constraints imposed by hermiticity, parity and time-reversal, reads

$V_{\lambda \lambda^{\prime}}^{\mu}=\frac{1}{(P n)} \epsilon_{\alpha}^{*}\left(p^{\prime}, \lambda^{\prime}\right) \mathcal{V}^{\mu, \alpha \beta}(P, \Delta, n) \epsilon_{\beta}(p, \lambda)$

with

$$
\begin{aligned}
\mathcal{V}^{\mu, \alpha \beta}(P, \Delta, n) \\
=g^{\alpha \beta}\left[P^{\mu} F_{1}+\Delta^{\mu} F_{2}+\frac{M^{2} n^{\mu}}{(P n)} F_{3}\right] \\
+\frac{\Delta^{\alpha} \Delta^{\beta}}{M^{2}}\left[P^{\mu} F_{4}+\Delta^{\mu} F_{5}+\frac{M^{2} n^{\mu}}{(P n)} F_{6}\right] \\
+\frac{M^{2} n^{\alpha} n^{\beta}}{(P n)^{2}}\left[P^{\mu} F_{7}+\Delta^{\mu} F_{8}+\frac{M^{2} n^{\mu}}{(P n)} F_{9}\right] \\
+\frac{n^{\{\alpha} \Delta^{\beta\}}}{(P n)}\left[P^{\mu} F_{10}+\Delta^{\mu} F_{11}+\frac{M^{2} n^{\mu}}{(P n)} F_{12}\right] \\
+\frac{n^{[\alpha} \Delta^{\beta]}}{(P n)}\left[P^{\mu} F_{13}+\Delta^{\mu} F_{14}+\frac{M^{2} n^{\mu}}{(P n)} F_{15}\right] \\
+g^{\mu\{\alpha}\left[\Delta^{\beta\}} F_{16}+\frac{M^{2} n^{\beta\}}}{(P n)} F_{17}\right] \\
+g^{\mu[\alpha}\left[\Delta^{\beta]} F_{18}+\frac{M^{2} n^{\beta]}}{(P n)} F_{19}\right],
\end{aligned}
$$

and $F_{i}=F_{i}(x, \xi, t)$. Since $\mathcal{V}^{\mu, \alpha \beta}(P, \Delta, n)$ must be invariant under a rescaling of the lightlike direction $n \mapsto \alpha n$, factors of $M /(P n)$ appear whenever necessary. Additional factors of the hadron mass $M$ have also been included to keep GPDs dimensionless. The relation between $F_{i}$ and the standard GPD basis $H_{i}, G_{i}$ of (40) can easily be obtained by projection onto the twist- 2 and 3 parts. 


\section{References}

1. E. Leader, C. Lorcé, Phys. Rept. 541, 163 (2014). arXiv:1309.4235 [hep-ph]

2. M.V. Polyakov, P. Schweitzer, Int. J. Mod. Phys. A 33, 1830025 (2018). arXiv:1805.06596 [hep-ph]

3. E. Cartan, Ann. Sci. Ecole Norm. Sup. 40, 325 (1923)

4. M.V. Polyakov, Phys. Lett. B 555, 57 (2003). arXiv:hep-ph/0210165 [hep-ph]

5. C. Lorcé, Eur. Phys. J. C 78, 120 (2018a). arXiv:1706.05853 [hep$\mathrm{ph}]$

6. C. Lorcé, L. Mantovani, B. Pasquini, Phys. Lett. B 776, 38 (2018). arXiv:1704.08557 [hep-ph]

7. M.V. Polyakov, A.G. Shuvaev, (2002). arXiv:hep-ph/0207153 [hep-ph]

8. C. Lorcé, H. Moutarde, A.P. Trawiński, Eur. Phys. J. C 79, 89 (2019). arXiv:1810.09837 [hep-ph]

9. M.V. Polyakov, H.-D. Son, JHEP 09, 156 (2018). [JHEP 18, 156 (2020)]. arXiv:1808.00155 [hep-ph]

10. I.V. Anikin, (2019). arXiv:1902.00094 [hep-ph]

11. J. Hudson, P. Schweitzer, Phys. Rev. D 96, 114013 (2017). arXiv: 1712.05316 [hep-ph]

12. S. Kumano, Q.-T. Song, O.V. Teryaev, Phys. Rev. D 97, 014020 (2018). arXiv:1711.08088 [hep-ph]

13. K. Tanaka, Phys. Rev. D 98, 034009 (2018). arXiv:1806.10591 [hep-ph]

14. C. Alexandrou, M. Constantinou, K. Hadjiyiannakou, K. Jansen, C. Kallidonis, G. Koutsou, A. Vaquero Avilés-Casco, C. Wiese, Phys. Rev. Lett. 119, 142002 (2017). arXiv:1706.02973 [hep-lat]

15. Y.-B. Yang, M. Gong, J. Liang, H.-W. Lin, K.-F. Liu, D. Pefkou, P. Shanahan, Phys. Rev. D 98, 074506 (2018a). arXiv:1805.00531 [hep-lat]

16. Y.-B. Yang, J. Liang, Y.-J. Bi, Y. Chen, T. Draper, K.-F. Liu, Z. Liu, Phys. Rev. Lett. 121, 212001 (2018b). arXiv:1808.08677 [hep-lat]

17. P.E. Shanahan, W. Detmold, Phys. Rev. D 99, 014511 (2019). arXiv:1810.04626 [hep-lat]

18. P. E. Shanahan, W. Detmold (2018). arXiv: 1810.07589 [nucl-th]

19. T. Horn, C.D. Roberts, J. Phys. G 43, 073001 (2016). arXiv:1602.04016 [nucl-th]

20. R.L. Jaffe, A. Manohar, Phys. Lett. B 223, 218 (1989)

21. Z. Abidin, C.E. Carlson, Phys. Rev. D 77, 095007 (2008). arXiv:0801.3839 [hep-ph]

22. W. Detmold, D. Pefkou, P.E. Shanahan, Phys. Rev. D 95, 114515 (2017). [Erratum: PhysRevD.99.019904]. arXiv:1703.08220 [heplat]

23. W. Boeglin, M. Sargsian, Int. J. Mod. Phys. E 24, 1530003 (2015). arXiv:1501.05377 [nucl-ex]

24. A. Airapetian et al., (HERMES), Phys. Rev. Lett. 95, 242001 (2005). arXiv:hep-ex/0506018 [hep-ex]

25. P. Hoodbhoy, R.L. Jaffe, A. Manohar, Nucl. Phys. B 312, 571 (1989)

26. N.N. Nikolaev, W. Schafer, Phys. Lett. B 398, 245 (1997). [Erratum: Phys. Lett.B407,453(1997)]. arXiv:hep-ph/9611460 [hep-ph]

27. K. Bora, R.L. Jaffe, Phys. Rev. D 57, 6906 (1998). arXiv:hep-ph/9711323 [hep-ph]

28. J. Edelmann, G. Piller, W. Weise, Z. Phys. A 357, 129 (1997). arXiv:nucl-th/9701026 [nucl-th]

29. G.A. Miller, Phys. Rev. C 89, 045203 (2014). arXiv:1311.4561 [nucl-th]

30. W. Cosyn, Y.-B. Dong, S. Kumano, M. Sargsian, Phys. Rev. D 95, 074036 (2017). arXiv:1702.05337 [hep-ph]

31. D. Boer, S. Cotogno, T. van Daal, P.J. Mulders, A. Signori, Y.-J. Zhou, JHEP 10, 013 (2016). arXiv:1607.01654 [hep-ph]

32. S. Cotogno, T. van Daal, P.J. Mulders, JHEP 11, 185 (2017). arXiv:1709.07827 [hep-ph]
33. W. Detmold, P.E. Shanahan, Phys. Rev. D 94, 014507 (2016). [Erratum: Phys. Rev.D95,no.7,079902(2017)]. arXiv:1606.04505 [heplat]

34. F. Winter, W. Detmold, A.S. Gambhir, K. Orginos, M.J. Savage, P.E. Shanahan, M.L. Wagman, Phys. Rev. D 96, 094512 (2017). arXiv:1709.00395 [hep-lat]

35. K. Slifer, Proceedings, Tensor Polarized Solid Target Workshop: Newport News, USA, March 10-12, 2014. J. Phys. Conf. Ser. 543, 012003 (2014)

36. B.R. Holstein, Phys. Rev. D 74, 084030 (2006). arXiv:gr-qc/0607051 [gr-qc]

37. S.K. Taneja, K. Kathuria, S. Liuti, G.R. Goldstein, Phys. Rev. D 86, 036008 (2012). arXiv:1101.0581 [hep-ph]

38. A. Accardi et al., Eur. Phys. J. A 52, 268 (2016). arXiv:1212.1701 [nucl-ex]

39. D. Kikoła, M.G. Echevarria, C. Hadjidakis, J.-P. Lansberg, C. Lorcé, L. Massacrier, C.M. Quintans, A. Signori, B. Trzeciak, Few Body Syst. 58, 139 (2017). arXiv:1702.01546 [hep-ex]

40. A. Airapetian et al., (HERMES), Nucl. Phys. B 829, 1 (2010). arXiv:0911.0095 [hep-ex]

41. A. Airapetian et al., (HERMES), Nucl. Phys. B 842, 265 (2011). arXiv: 1008.3996 [hep-ex]

42. M. Mazouz et al., (Jefferson Lab Hall A), Phys. Rev. Lett. 118 222002 (2017). arXiv:1702.00835 [hep-ex]

43. A. Camsonne, M. Carmignotto, E. Fuchey, R. Montgomery, A. Puckett, Z. Ye, Z. Ye, et al., (Jefferson Lab Hall A SBS), Deeply virtual compton scattering using the tagged deeply inelastic scattering experimental setup (2018). LOI12-18-002

44. W. Armstrong et al. (2017). arXiv:1708.00888 [nucl-ex]

45. P. Achard et al., (L3),Phys. Lett. B 568, 11 (2003). arXiv:hep-ex/0305082 [hep-ex]

46. I.V. Anikin, B. Pire, O.V. Teryaev, Phys. Rev. D 69, 014018 (2004). arXiv:hep-ph/0307059 [hep-ph]

47. I.V. Anikin, B. Pire, O.V. Teryaev, Phys. Lett. B 626, 86 (2005). arXiv:hep-ph/0506277 [hep-ph]

48. C. Lorcé, Eur. Phys. J. C 78, 785 (2018b). arXiv:1805.05284 [hep$\mathrm{ph}]$

49. W. Florkowski, R. Ryblewski, (2018). arXiv:1811.04409 [nucl-th]

50. I.I. Balitsky, V.M. Braun, Nucl. Phys. B 311, 541 (1989)

51. C. Lorcé, JHEP 08, 045 (2015). arXiv:1502.06656 [hep-ph]

52. T. Frederico, E.M. Henley, S.J. Pollock, S. Ying, Phys. Rev. C 46, 347 (1992)

53. E.R. Berger, F. Cano, M. Diehl, B. Pire, Phys. Rev. Lett. 87, 142302 (2001). arXiv:hep-ph/0106192 [hep-ph]

54. H. Joos, G. Kramer, Z. Phys. 178, 542 (1964)

55. D. Zwanziger, Phys. Rev. B 137, 1535 (1965)

56. B.L.G. Bakker, E. Leader, T.L. Trueman, Phys. Rev. D 70, 114001 (2004). arXiv:hep-ph/0406139 [hep-ph]

57. K.-F. Liu, C. Lorcé, Eur. Phys. J. A 52, 160 (2016). arXiv:1508.00911 [hep-ph]

58. K.R. Symon, Mechanics, 3rd edn. (Addison-Wesley Pub. Co, Reading, MA, 1971)

59. H. Goldstein, Mechanics, 2nd edn. (Addison-Wesley, Reading, MA, 1980)

60. R.L. Jaffe, A. Manohar, Nucl. Phys. B 337, 509 (1990)

61. X.-D. Ji, Phys. Rev. Lett. 78, 610 (1997). arXiv:hep-ph/9603249 [hep-ph]

62. X.-D. Ji, J. Phys. G G24, 1181 (1998). arXiv:hep-ph/9807358 [hep$\mathrm{ph}]$

63. M.V. Polyakov, C. Weiss, Phys. Rev. D 60, 114017 (1999). arXiv:hep-ph/9902451 [hep-ph]

64. H.-D. Son, H.-C. Kim, Phys. Rev. D 90, 111901 (2014) arXiv: 1410.1420 [hep-ph]

65. W. Cosyn, A. Freese, B. Pire, Phys. Rev. D 99, 094035 (2019). arXiv:1812.01511 [hep-ph] 
66. M. Penttinen, M.V. Polyakov, A.G. Shuvaev, M. Strikman, Phys. Lett. B 491, 96 (2000). arXiv:hep-ph/0006321 [hep-ph]

67. M. Diehl, Phys. Rept. 388, 41 (2003). arXiv:hep-ph/0307382 [hep$\mathrm{ph}]$

68. I.V. Anikin, R.S. Pasechnik, B. Pire, O.V. Teryaev, Eur. Phys. J. C 72, 2055 (2012). arXiv:1112.1849 [hep-ph]

69. D.V. Kiptily, M.V. Polyakov, Eur. Phys. J. C 37, 105 (2004). arXiv:hep-ph/0212372 [hep-ph]

70. Y. Hatta, S. Yoshida, JHEP 10, 080 (2012). arXiv:1207.5332 [hep$\mathrm{ph}]$

71. A. Bacchetta, P.J. Mulders, Phys. Rev. D 62, 114004 (2000). arXiv:hep-ph/0007120 [hep-ph]

72. X. Artru, M. Mekhfi, Z. Phys. C 45, 669 (1990)

73. A.V. Efremov, O.V. Teryaev, Sov. J. Nucl. Phys. 36, 557 (1982). [Yad. Fiz.36,950(1982)]

74. A.V. Efremov, O.V. Teryaev, in Dubna Deuteron - 93 (1994). arXiv:hep-ph/9910555 [hep-ph]
75. M.V. Polyakov, B.-D. Sun, (2019). arXiv:1903.02738 [hep-ph]

76. X.-D. Ji, R.F. Lebed, Phys. Rev. D 63, 076005 (2001). arXiv:hep-ph/0012160 [hep-ph]

77. S. Liuti, in Talk at INT Workshop (February 6-17, 2012). http:// www.int.washington.edu/talks/WorkShops/int_12_49W/People/ Liuti_S/Liuti.pdf

78. S. Liuti, in 23rd International Symposium on Spin Physics (SPIN 2018) Ferrara, Italy, September 10-14, 2018 (2019) arXiv:1903.08760 [hep-ph]

79. J. Krause, J. Math. Phys. 18, 889 (1977)

80. D.E. Fahnline, Am. J. Phys. 50, 818 (1982). https://doi.org/10. $1119 / 1.12748$

81. C. Lorcé, Phys. Rev. D 97, 016005 (2018). arXiv: 1705.08370 [hep$\mathrm{ph}]$

82. W.N. Polyzou, W. Glöckle, H. Witala, Few Body Syst. 54, 1667 (2013). arXiv:1208.5840 [nucl-th] 